\title{
RFP: Request for Pedagogy
}

IRENE HWANG

University of Michigan
As the professional reality of architectural practice has radically transformed in the last decade, the teaching of professional practice as a core course has remained static for four times as long. Even while current practice explodes into many different innovative models and methods, the teaching of professional practice has hardly budged. We must turn our focus to a new tertiary, the territory between the historical binary of the scholarly pursuit of the academy and the practical work of the profession.

To that end, this paper presents a new mindset for teaching professional practice by unpacking piloted methods and concepts through five compact case studies:

1) First Day of Class: Setting the Tone for Engagement

2) Syllabus: The Importance of Transparency

3) Writing The Syllabus: The Importance of Transparency

4) Curricular Value: Why Credits Matter

5) RFP: Request for Pedagogy

\section{INTRODUCTION}

'McKim would indicate to the draftsman where to draw lines and correct them: 'He looked at them for a long time and then said "Just take out that middle line and move it up a little...No, put it back where it was-perhaps a little lower"... it was quite a job to erase and remake the lines smeared in the process, and to repeat that sort of thing for hours on end was hard on the nerves of anyone.'

-H. Van Buren Magonigle, Pencil Points, 1934

Though Magonigle describes an experience one hundred years old, such interactions remain widespread in the architecture discipline of today. This autocratic structure, otherwise instituted as the Beaux-Arts model, was for generations an effective, highly competitive model to ensure the rise of the best work from a group of like-minded and similarly trained individuals.

Today, as architectural practice rapidly diversifies through globalization and technological advances, we face a critical demand for an entirely new mindset when it comes to architectural education. The agility to move between multiplying roles, changing responsibilities, and expanding opportunities is now at a scale far beyond the capacities of one person. The top-down Beaux-Arts mindset, which prioritizes efficiency and competition in the interest of the best answer (above all else) cannot support architectural education, nor architectural practice as we need them today. The meaning of success has radically changed: to inspire and motivate others is far more valuable than maximizing individual productivity (whether singular or aggregated, creative or analytic).

What are the next generation of skills, expertise, and intellectual frameworks necessary to create this new mindset? While we should not rehaul the curriculum in its entirety, where we begin change is in how to evolve the teaching of professional practice in the academy.

\section{CASE I. FIRST DAY OF CLASS: SETTING THE TONE FOR ENGAGEMENT}

The first impression one strikes with students is not to be underestimated. Especially in the realm of professional practice, where students may already have a bias toward the class as non-experimental, rote, and non-creative, it has been particularly important in the restructuring of the requiredgraduate-level course, Arch 583, at the Taubman College, to reveal immediately, our pedagogical desire to rethink the culture of architectural thinking and making, as the core driving intention of such a class.

Whereas mentioned in the introduction, the Beaux-Arts model of teaching was ingeniously adept and effective at ensuring that the "best minds" were at work on a shared problem. While in centuries past, such a model resulted in a solution or outcome that was fitting for a more homogenous context, today's heterogenous and diverse, global context is no longer able to be satisfied with a single-minded view of anything Simply put, one man's answer is no longer a one-size-fits-all panacea to any number of mixed-gender, mixed-culture, or mixed-value situations.

To that end, for our first day of class, we don't speak of practice, but rather we introduce our students to our understanding of the unique context we find ourselves working in. We ask them to take a position on the culture of architectural production, both thinking and making, and propose to our students that they consider how to influence the evolution of that shared 

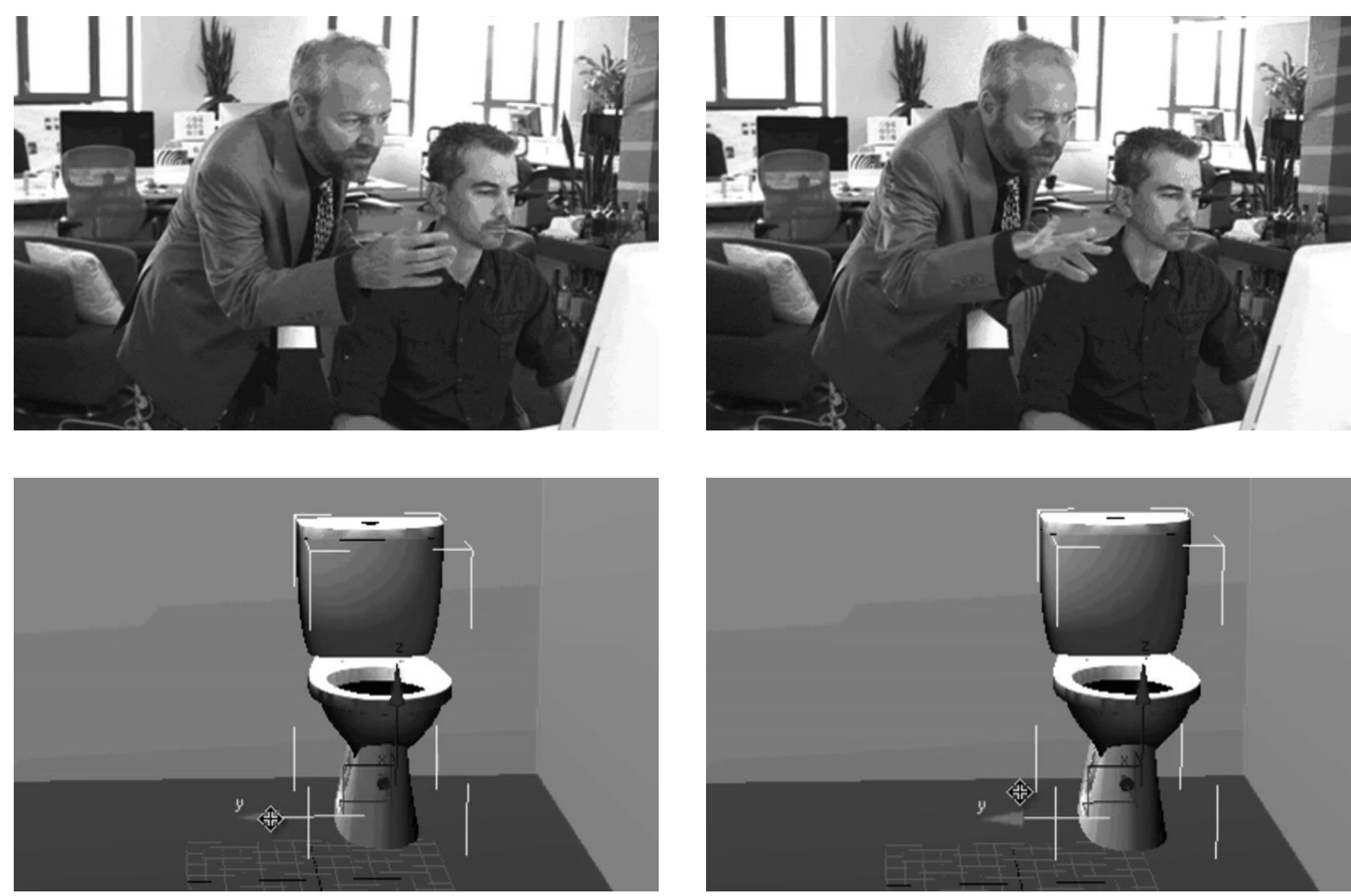

Figure 1-4. "Architect" gif on Giphy, showing an animated loop of an architect asking his employee to move a toilet, back and forth, left to right

culture. In particular, we start the course by first forming working groups of four or five students, and then, inspired by the inter-connectivity and interactivity of digital channels, students generate and share their work in real time, to the entire class, via Google Sheets. [Fig. 5]

Outside of the classroom, parallel initiatives also contribute to building the "first impression." In acknowledgement of the diversity and simultaneous importance of, one such initiative, in collaboration with Joana dos Santos, our chief diversity, equity and inclusion officer, we have invited all students to participate in sharing the phonetic pronunciation of their names, through the creation of name cards that they can bring to class and place on studio desks. For a visual community, the written card removes the awkwardness of repeated re-pronunciation and correction, as well as provide an easily accessed, written reference. This simple initiative, which hopes to understand the importance that names-as we wish them pronouncedhave a visceral and powerful connection to one's individual identity, has been incredibly well received by both faculty and students. [Fig. 6]

\section{CASE II. WRITING THE SYLLABUS: THE IMPORTANCE}

\section{OF TRANSPARENCY}

In writing the syllabus for a graduate-level, core course in the M.Arch-degree program, my co-teacher, Daniel Jacobs, and I began by dividing the course into three parts: 01 PRACTICE, 02 SERVICE, and 03 ENTREPRENEURSHIP. Each module is of equal value; each module has a companion, major project that asks students to ideate on where and how to use their architectural educations and to promote interleaved learning.

What does transparency mean for a syllabus? For us it meant first being self-reflective about our experiences in practice. What elements of the discipline are changing? What elements of the profession do we value as teachers and what did we want to convey to the students?

By restructuring and re-writing of the syllabus for the longstanding course, Arch 583 - Professional Practice, our work has brought about the creation of new insights, along with the development of new procedural methods for delivering interactive and meaningful teaching to students. Additionally, the act of creating and writing a course and syllabus has been a pedagogic tool, to be used directly in the development of our scholarship and teaching. Moving forward, the writing of a new syllabus allows us to be analytical of past 


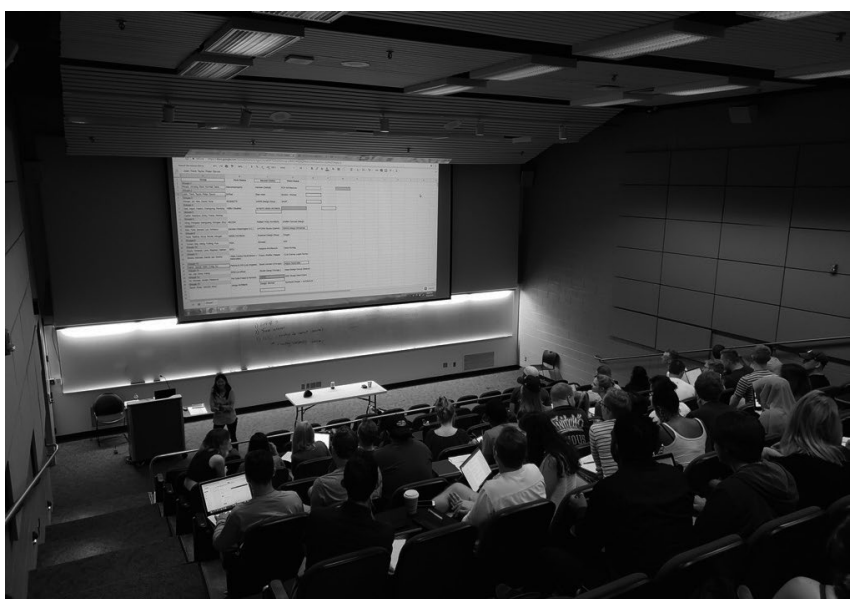

Figure 5. Dividing up into teams and topics, Day 1

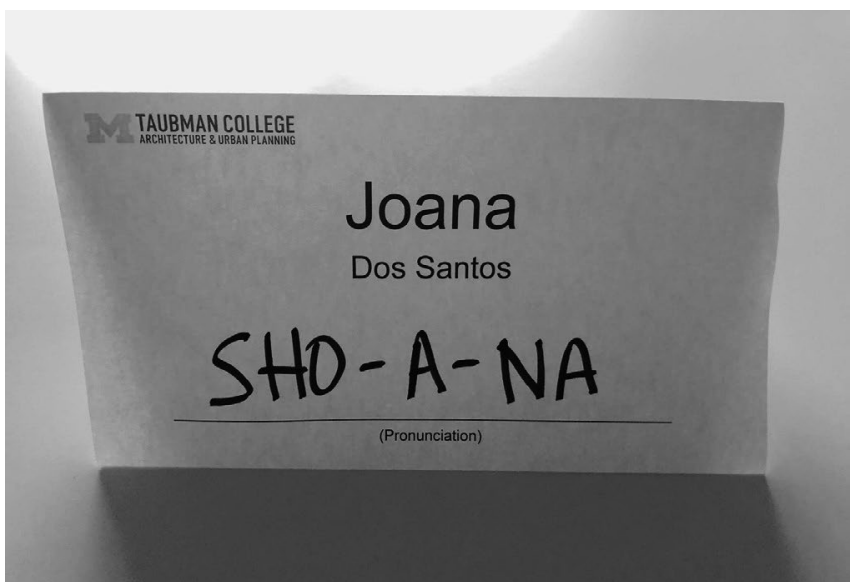

Figure 6. Phonetic-Naming Initiative

professional and academic experiences, as well as, gain new perspectives through autonomous learning, a fundamental component of academic work and professional development. Our personal, pedagogic contexts are revealed to our students through the syllabus.

In such, the course examines the complexities of the discipline, intertwined and complicit with broader global and local economic conditions, labor markets, political issues, and cultural representation. 01 PRACTICE grapples with the foundational ideas of what it means to be part of a "profession" and what the privileges and responsibilities such a distinction implies. To do this, the course interrogates and contextualize the historical, practical, ethical, organizational, legal, financial, social, and technological conditions embedded in the practice of architecture. 02 SERVICE explores the mechanisms and modalities that architects can use to promote a more just, equitable, and environmentally conscious discipline. As stewards of the built environment, how can architects use their expertise towards new forms of public service and participation? Students propose a service-minded project to create social impact for their hometowns, presenting their work through a two-minute video. 03 ENTREPRENEURSHIP will engage with the emerging technologies, platforms, networks, intellectual property, and alternative business models changing the practice of architecture. Students will research and propose an AEC start-up, capitalizing on the skills of the architect and critically interrogating market conditions. Through lectures, workshops, case studies, readings, research, and projects, the course cover these essential elements and obstacles of the profession in order that students can critically examine the potential agency of the architect.

ARCH 583 has been designed as a space for civic participation, to expand the scope of influence of the architect towards a better future for policy, culture, technology, humanity, and the built environment.

\section{CASE III. CURRICULAR VALUE: WHY CREDITS MATTER}

As we transition away from process-based work (e.g., working to optimize and perfect a repetitive, predictable process) and toward more and more project-based work (e.g., creating an entirely new outcome via a collaborative enterprise), the nature of how we organize, to work and create, demands moving beyond traditional learning and apprenticeship models, or the master-led structure, whose origins lie in the widespread emulation and adoption of the French École des Beaux-Arts teaching model throughout American architectural education.

Before establishing the first American academic program for architecture at MIT in 1865, its director, William Robert Ware, studied École methods for three years before the department began instruction in 1868. Over the next three decades, as universities such as Michigan, Cornell, Harvard, and Columbia created their own architecture programs, nine of the ten were led by American alumni or teachers from the École. In the Beaux-Arts atelier, all exercises began with a project problem. Afterward, each student worked on his own solution, with "no assistance and guardians patrolled the corridors to see you received none." [- Joseph $\mathrm{H}$. McGuire, enrolled at the École in 1889] A benefit of this model encouraged "many solutions of the same project" [-Julian Clarence Levi, École alumni] and gave rise to case study teaching. For centuries, the Beaux-Arts model created an effective, highly competitive structure that ensured the best work among a group of like-minded and similarly trained individuals would be identified and recognized.

Increasingly, the primary challenge facing architecture design professionals is how to collaborate on larger projects at larger and larger distances. The management of these distances, which take on a variety of forms (language, time, culture, traditions, preferences, climate, supply chains, building methods among many, many others) is very complex, even for the most modest of architecture projects. The outcome has been a critical demand for highly collaborative work across all levels of the architecture discipline and profession. 


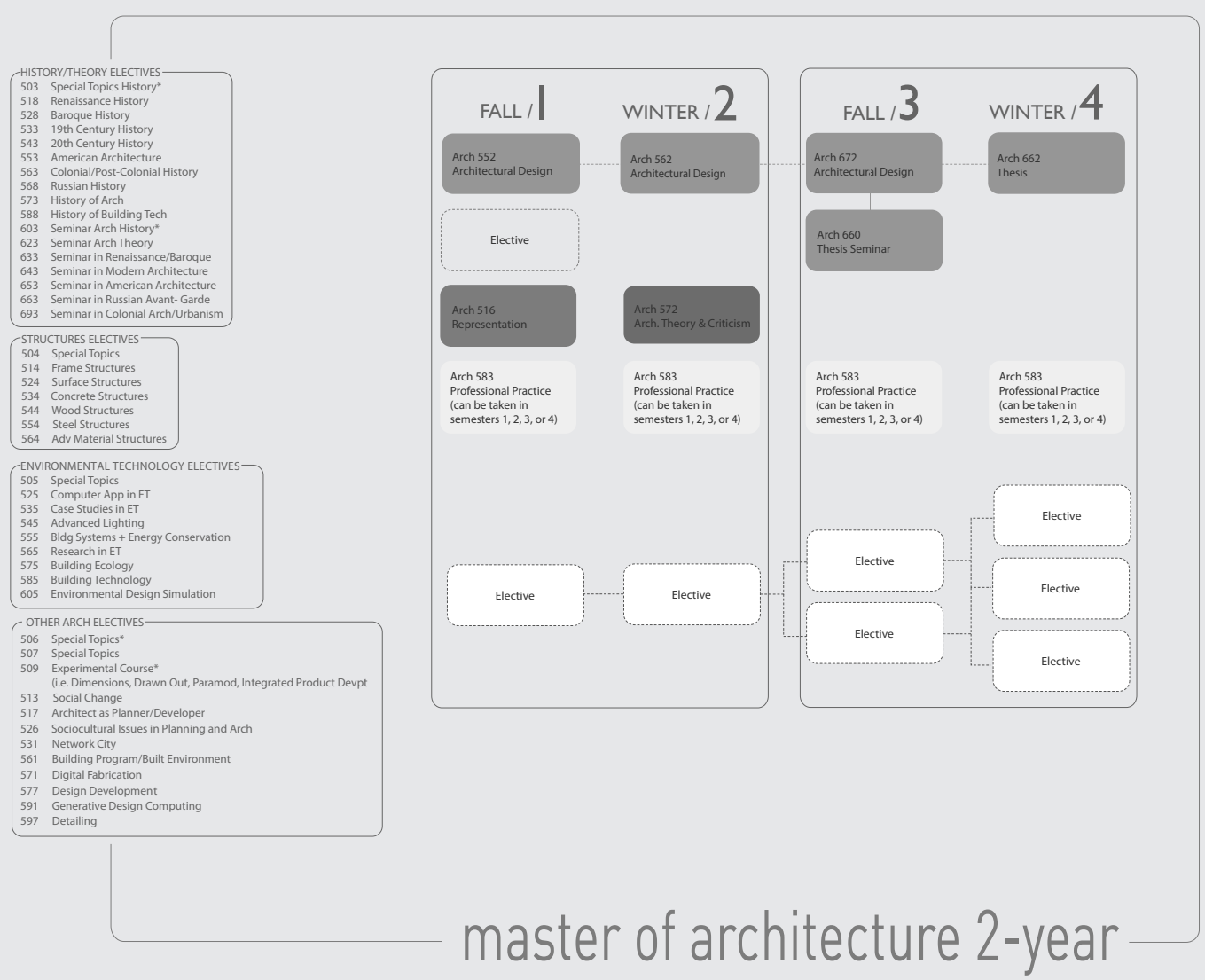

Figure 7 (above): Revised Arch 583, Professional Practice location within the M.Arch, two-year curriculum

While diversity is a welcome result of globalization, the threats of miscommunications and misunderstandings also multiply due to these increased distances. While new technologies and processes bring about speed and efficiencies, they have introduced far more complex systems and sets of relationships for an architect to navigate and manage. These systems revise the socio-economic landscape so rapidly that relying on previous factors of success (e.g., homogenization of work; standardized, repetitive tasks; strict adherence to set instructions) cannot keep up with the demands of more complex work and more complex teams of specialists working on projects together.

Though our students are working more and more in groups, they continue to report difficulties: spending too much time coordinating among members; feeling stuck picking up the slack of reluctant collaborators. Many would rather sidestep the complexities and demands of collaboration by having less team projects, choosing smaller groups, or opting to work alone. We can't fully capitalize on the benefits of diversity without imbuing in our students a shift in the persistent master-based organizational structure of architectural design and delivery work.

In Case III, we have re-framed the nature of the group project, by increasing responsiveness and horizontality. By having three, major, equally weighted projects per semester, our curricular intention is to ensure that students consider all to be of equal importance. Having previously encouraged students to selforganize, we've seen the limits of that structure and now rely on a completely randomized process to devise student groups. Moreover, we noted another hurdle to successful group work: group fatigue. By ensuring that each project not only refreshes the class's thinking, we also concurrently refresh the collaborative chemistry by creating entirely new groups for each project. Thus, we gain responsiveness by providing students with the opportunity to practice-several times a semester-the operation of starting a new group, building social connection within that new group, as well as understanding how the group can arrive at a mutual understanding in why and how to be creative, innovative, as well as, efficient and productive. By not dictating how to come together or instituting instruction on how to work, the students are able to devise their own teaming mechanisms, which they are able to then tweak and improve upon in future group settings, both in and beyond the classroom.

\section{CASE IV. CURRICULAR VALUE: CREDITS MATTER}

It is taken for granted that professional practice is, for the most part, situated at the end of an architectural curriculum. In contrast to both technical (e.g., construction, structures) 


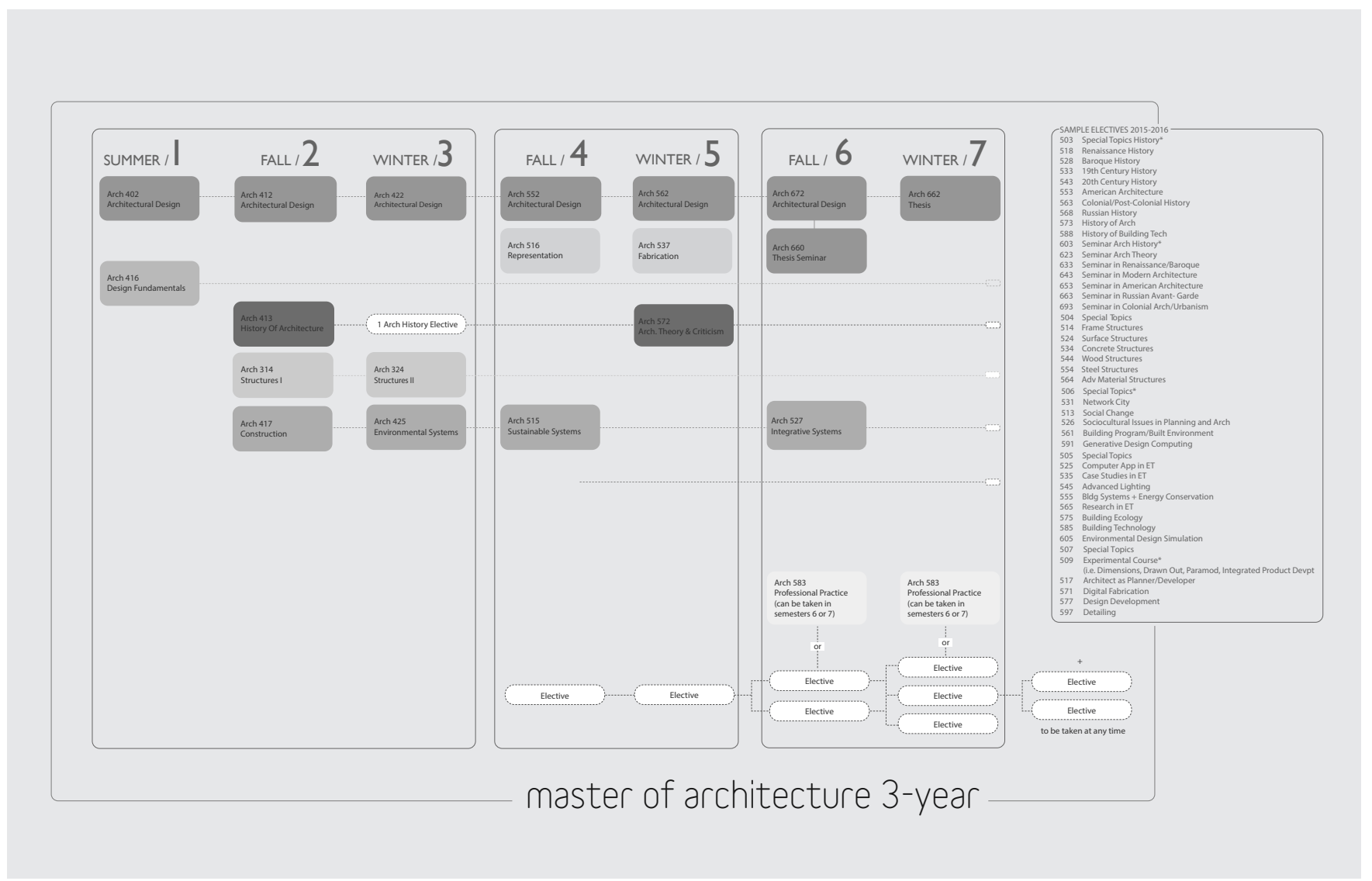

Figure 8 (below) : Legacy position, in yellow, of Arch 583 at the end of an M.Arch curriculum

and experimental (e.g., studio, theory) courses, the singular professional practice course has held onto its function as a bridge course, a way to impart information as a professional introduction to almost-graduated students: its primary function, to deliver information to students and familiarize them with the next steps to licensure and an exclusive professional occupation in building design and delivery. It has been, frankly, an introductory course that was in deep contrast to the advanced coursework that would occupy the majority of a final-semester graduate student's schedule. Students prioritized their advanced coursework, in that they were far more engaged in the content of those classes, and displayed notably less involvement in their study and learning of professional practice.

Furthermore, in light of our professional colleagues working and making impact in other fields such as policy, technology, and business, we have innovated Arch 583 to support our students in their quest to take their architectural educations into these other realms. In our minds, pedagogic innovation does not occur in isolation. To ensure that students can benefit from our new curricular positioning of Arch 583, we have worked with our registrar, Stacey Shimones, to not only reposition the course within the curriculum-as a foundational, introductory course-but also to ensure that students are able to attend, conflict-free from other earlydegree, required courses. [Figs. 7-8]

By introducing an expanded understanding of professional practice to students at the early parts of their degree coursework, there has been a resultant increase in the engagement and participation of our students. For example, in some group projects, we have even seen some individuals voluntarily participate across groups to contribute to other teams' work, giving their time and efforts to work that is not part of their own graded submissions.

Finally, as with the writing of the syllabus, we have been explicit about the expectation of a 3 -credit-unit course at the University of Michigan (three hours in class + six to nine hours, outside of class), as well as, our request that students consciously assign coursework prioritization (in terms of time and attention) not only in Arch 583, but also across their entire course load. Our position is that we all receive values conveyed via a real, albeit, non-visible curriculum, and that to best take advantage of our education, we find ways to consciously understand and/or counteract our biases. 


\section{Question 4: What keeps your students up at night?

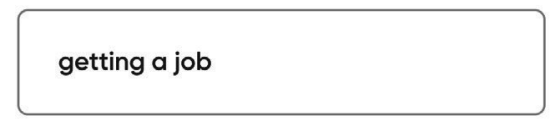 \\ Missing the party on the beach \\ Mostly studio work! \\ Working in assessments with studio. Studio takes priority. \\ Many outside factors, including concerns about family, work \\ Not pro practice \\ How to balance school with providing for self/family \\ They don't get specific enough guidance on time management. Faculty need to help students understand appropriate limits. It's an ongoing problem. \\ Studio studio studio stress studio stress bad romance studio studio stress stress studio \\ Getting a job and keeping it. \\ Balancing life, work and school \\ Rhino \\ Wondering how to find the right answer \\ What is academia vs practice? \\ Requirements, work life balance, are}

Figure 9 (Top): Figure 9 (Above) : Session participants responses via Mentimeter interactive presentation software;

\section{CASE V. RFP: REQUEST FOR PEDAGOGY}

At the close of my presentation at the 2019 Less Talk - More Action conference, Case $V$ was the opportunity for educators and administrators across accredited programs to share their perspectives and responses to the four, following questions [Fig. 9-10] :

Question 1. What is missing from your school's pro-practice curriculum?

"Entrepreneurship, and more broadly training students how to have agency in the built environment. How can they be more than simply service providers waiting for clients to come to them with needs?"

"Preparation for the A.R.E."

"Enthusiasm. As well, as conversations about technology and its impact on our discipline and other modes of working that are not the traditional firm route."

Question 2. What is working in your school's pro practice curriculum?

"We don't know because pro practice is isolated from the rest of the curriculum and never discussed in faculty meetings."

"We heavily cover public interest design and non-standard models of practice and have a new undergrad pro practice class on collaboration and leadership."

"Good overview of traditional modes of practice."
Question 3. What is not working in your school's pro practice curriculum?

"Not integrating with the rest of the curriculum."

"Outdated ideas about practice. Not keeping up with relevant disciplinary shifts. Lacking info about diversity."

"Link to immediately relevant info for students starting a practice right out of school."

\section{Question 4. What keeps your students up at night?}

"Studio studio studio stress studio stress bad romance studio studio stress stress studio."

"Pressure from studio instructors who assign too much work, fear of failing or even just getting a "C," anxiety, depression, fear of being shamed in public reviews."

"Getting a job."

As evidenced by this sample of responses from our colleagues, our personal and pedagogical experiences and ideas on professional practice teaching and curriculum are messy and open ended. What this feedback has meant for me, is the realization that there is no singular path forward that encompass the diversity of ways that professional practice teaching and curriculum can contribute to our students' educations. It seems that our work in advancing the pedagogy of teaching practice is able to draw from a multitude of perspectives and 

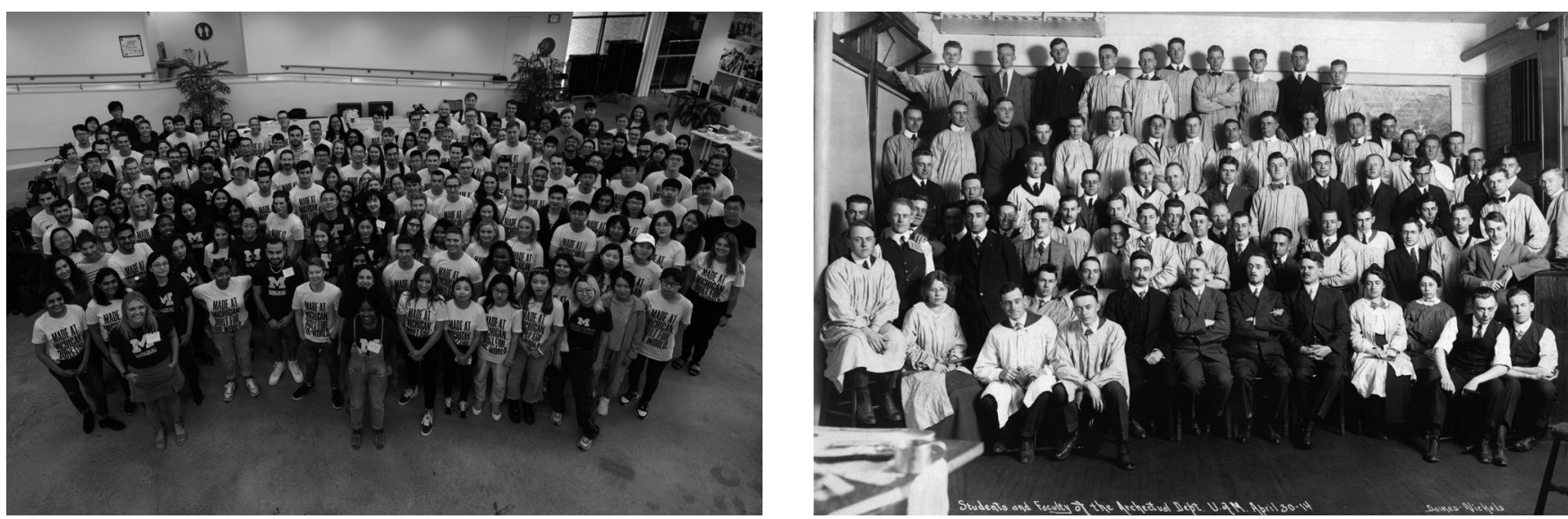

Figures 10-11 (Above) : Changing face of student cohort, University of Michigan, Taubman College, 1914 and 2019 incoming classes. Images courtesy of Taubman College and University of Michigan Bentley Historical Library

trajectories, something that it has not been able to take advantage of until now. Not only is our profession diversifying, but so is our capacity to teach it.

\section{CONCLUSION}

I left the conference with an incredible level of optimism: for what we have accomplished; for what we will be able to do; for the future of our students who have chosen to undertake an education and practice in architecture-in all the many ways that our discipline is now able to operate and contribute. 